\title{
Attentional selectivity, automaticity, and self-efficacy predict simulator-acquired skill transfer to the clinical environment
}

Nicholas E. Anton, $\mathrm{MS}^{1}$, Tomoko Mizota, $\mathrm{MD}^{1}$, Lava Timsina, $\mathrm{PhD}^{1}$, Jake Whiteside ${ }^{1}$, Erinn Myers, $\mathrm{MD}^{2}$, Dimitrios Stefanidis, $\mathrm{MD}, \mathrm{PhD}^{1}$

1. Department of Surgery, Indiana University School of Medicine

2. Carolinas Simulation Center, Carolinas Healthcare System

Correspondence concerning this article should be addressed to:

Dimitrios Stefanidis, MD, PhD, FACS, FASMBS, FSSH

Vice Chair of Education

Chief, MIS/bariatric Surgery

Department of Surgery

Indiana University School of Medicine

545 Barnhill Dr, EH 130

Indianapolis, IN 46202

Tel: (317) 278-1116

Email: dimstefa@iu.edu

\section{Funding}

This work was funded by the Agency for Healthcare Research and Quality (AHRQ) [grant number R18HS022080]. The AHRQ was not involved in any element of the study design, manuscript preparation, or in the decision to submit the article for publication.

This work was presented at a concurrent oral session during the annual Association for Surgical Education meeting in Austin, TX on 05/01/2018.

\begin{abstract}
Introduction

Several studies demonstrated that simulator-acquired skill transfer to the operating room is incomplete. Our objective was to identify trainee characteristics that predict the transfer of simulator-acquired skill to the operating room.
\end{abstract}

\section{Methods}

Trainees completed baseline assessments including intracorporeal suturing (IS) performance, attentional selectivity, self-reported use of mental skills, and self-reported prior clinical and simulated laparoscopic experience and confidence. Residents then followed proficiency-based laparoscopic skills training, and their skill transfer was assessed on a live-anesthetized porcine model. Predictive characteristics for transfer test performance were assessed using multiple linear regression.

\section{Results}

Thirty-eight residents completed the study. Automaticity, attentional selectivity, resident perceived ability with laparoscopy and simulators, and post-training IS performance were predictive of IS performance during the transfer test.

\section{Conclusions}

Promoting automaticity, self-efficacy, and attention selectivity may help improve the transfer of simulator-acquired skill. Mental skills training and training to automaticity may therefore be valuable interventions to achieve this goal.

Keywords Surgery, simulation, stress, mental skills, self-efficacy 


\section{Short Summary}

The transfer of simulator-acquired surgical skill to the clinical environment is incomplete, thus, the purpose of the current study was to assess which trainee characteristics predict the transfer of simulator-acquired laparoscopic skill to the operating room. Residents' automaticity, perceived ability in laparoscopic surgery and perceived ability on simulators, and attentional selectivity predict transfer of simulator-acquired suturing skill to suturing performance on a live porcine Nissen model. Mental skills training and training to automaticity may ensure the more complete transfer of trainees' simulator-acquired skill to the clinical environment. 


\section{Introduction}

Surgical education has significantly changed in the last 20 years, as duty hour restrictions have led to reduced operative exposure and diminished autonomy for surgical residents. ${ }^{1,2}$ Residents are afforded fewer opportunities to develop technical skill than previous generations of surgeons. Surgical simulation training has emerged as a potential method to afford surgical trainees an opportunity to develop surgical skills in a safe environment. A recent literature review on the various applications of surgical simulation affirms that simulation-based surgical skills training promotes robust skill acquisition for trainees. ${ }^{3}$

Given this existing need and benefits provided by simulation medical schools and surgical departments have readily adopted surgical simulation training programs with medical students and residents. ${ }^{4,5}$ However, several studies demonstrated that in spite of meeting requisite expert-level proficiency benchmarks in the simulation environment, the transfer of simulatoracquired skill to the clinical environment is incomplete. ${ }^{6,7}$ Additional research in this area has identified that the clinical environment may cause increased stress which prevents the complete transfer of simulator-acquired skill. ${ }^{8}$

Mental skills are psychological techniques that enable performers to optimize practice and performance in on-demand situations, can be utilized to manage stress and attention during practice and performance. ${ }^{9}$ The use of such skills that may help diminish their stress and optimize their performance may, thus, predict surgery residents' ability to perform in challenging and stressful performance situations. Specifically, mental imagery, which is synonymous with mental rehearsal or mental practice, refers to an individual's ability to mentally create or recreate an experience in the absence of physical stimuli. ${ }^{10}$ Previous research has demonstrated that mental imagery use is associated with enhanced surgical performance through greater surgical 
skill acquisition and performance, confidence, knowledge, and teamwork. ${ }^{10}$ Also, attentional selectivity refers to one's ability to concentrate on task-relevant stimuli and disregard taskirrelevant stimuli, and surgeons' ability to selectively attend to relevant stimuli may be indicative of expertise. $^{11}$

The purpose of the current study was to identify baseline trainee characteristics that predict simulator-acquired skill transfer to the clinical environment.

\section{Methods}

Data from voluntary General Surgery and Obstetrics and Gynecology residents who previously participated in an Institutional Review Board-approved randomized controlled study ${ }^{12}$ across two institutions (Indiana University School of Medicine, Indianapolis, IN and Carolinas HealthCare System, Charlotte, NC) was analyzed.

\section{Baseline Testing}

Following informed consent, residents completed a baseline testing battery including their demographic characteristics, mental skills use, attention, trait and state anxiety, and laparoscopic performance (Fig. 1). Participants also reported their previous laparoscopic surgery experience (i.e., number of cases performed, assisted, observed as camera operator, and observed only), and perceived ability in laparoscopic surgery and on laparoscopic simulators (i.e., on a 20-point Visual Analog Scale) that we have utilized previously. ${ }^{13}$ Mental skills use in practice (i.e., surgical simulation) and performance (i.e., performing hands on patient care) settings was assessed using the Test of Performance Strategies- Version 3 (TOPS-3). ${ }^{14}$ The TOPS-3 is a 68item self-report instrument where participants rate their use of various mental skills such as mental imagery, attentional control, energy management, automaticity, use of self-talk, 
emotional control, goal setting, activation management (i.e., psyching up and relaxation techniques), and negative thinking on a 5-point Likert scale. The TOPS-3 was modified by the study team for use with surgeons (i.e., providing surgery-specific contextual information on how to approach answering questions) with permission from the authors. Subscales representing each participants' average use of mental skills in practice and performance situations can be extracted from the data and were done so to identify the predictive ability of specific mental skills.

Additionally, mental imagery was assessed using the Mental Imagery Questionnaire (MIQ) and the Sports Imagery Questionnaire (SIQ). The MIQ is an 8-item self-report measure of all areas of mental imagery related to laparoscopic surgery including how well users are able to engage in kinesthetic and visual imagery, the perceived effectiveness of engaging in mental imagery prior to laparoscopic performance, and their confidence to carry out a laparoscopic procedure. ${ }^{15}$ Users rate each item on a 7-point Likert scale, ranging from $1=$ "Not at all" to $7=$ "Very". The MIQ was developed for use with surgeons, and has been previously shown to have high reliability and face validity. ${ }^{15}$ The SIQ is a questionnaire widely used in the sport settings to assess an individual's effective use of imagery, which was adapted by the study team for use with surgeons with permission from the authors. ${ }^{16}$ Thirty statements depicting the use of imagery are rated on a 7-point Likert scale indicating how frequently the person uses imagery in the manner described (i.e., rarely to often). The SIQ yields scores on five factors that are classified as either cognitive or motivational in nature.

Residents' attentional selectivity was assessed with the D2 Test of Attention (D2), which is a written, timed test of selective attention. ${ }^{17}$ Users are asked to scan 14 lines with 47 of the letters "d" or "p" arranged with 1-4 dashes arranged above and/or below the letter, and are given 20 seconds per line to cross out each letter "d" with 2 total dashes. Concentration performance is 
the metric derived by subtracting the number of errors from the total number of correct possible items for each line and summing the cumulative total of each line, which reflects attentional selectivity. Participants' state and trait anxiety was also assessed at baseline using the State-Trait Anxiety Inventory-Full Version (STAI-F). ${ }^{18}$ The STAI-F is the most widely used self-report measure of anxiety in adults, and consists of 40-total items on two subscales which relate to how one feels in the moment (i.e., state) and how one generally deals with stress (i.e., trait). Laparoscopic performance was assessed using the Fundamentals of Laparoscopic Surgery (FLS) peg transfer, pattern cut, and intracorporeal suturing tasks. ${ }^{19}$ Training Following baseline testing, residents participated in eight, 45-minute sessions of proficiencybased laparoscopic skills training with coaching from experienced laparoscopic skills trainers over a period of approximately three to four months. These sessions aimed to train residents in peg transfer, pattern cut, and intracorporeal suturing. During these sessions, participants were coached on how to optimize their laparoscopic performance and instructed to attain the FLS proficiency guidelines for the aforementioned tasks, ${ }^{19}$ which included: moving all pegs from starting position to the opposite side of the board and back in 48 seconds or less on two consecutive repetitions and ten additional non-consecutive repetitions for peg transfer, removing the demarcated circle from the gauze pad with no cuts deviating from two millimeters of the demarcated line in 98 seconds or less on two consecutive repetitions for pattern cut, and completing intracorporeal suturing repetitions without errors in accuracy or knot security in 112 seconds or less on two consecutive repetitions and ten additional nonconsecutive repetitions. Due to logistical constraints, if residents were unable to achieve proficiency at the various tasks in a certain amount of time (i.e., up to two sessions for peg transfer and pattern cut, and 
intracorporeal suturing by the end of the eighth session), they were asked to proceed to the next task or allowed to participate in the transfer test. 


\section{Transfer test}

Following training, residents participated in a transfer test of suturing skill on a live porcine model. The porcine model's fundus was prepared for testing including the placement of two targets for participants' placement of the suture. The preparation of the model has been detailed extensively elsewhere. ${ }^{8}$ Participants were instructed to place three intracorporeal gastrogastric sutures on the porcine model's fundus using the same simulator-acquired technique and instrumentation as the FLS intracorporeal suturing task. However, unlike training, an attending surgeon drove the laparoscope and the operating room setup mimicked the human operating room (e.g., a $30^{\circ}$ laparoscope was used, with a laparoscopic tower setup, the porcine model was hooked up to a ventilator, and heart rate alarm sounds were audible). Suturing performance was evaluated utilizing a previously published formula: Performance Score $=600-$ Time to complete repetition (in seconds) $-[10 \mathrm{x}$ mm outside target area $]-[100 \mathrm{x}$ errors in knot security $(0=$ secure knot, 1 = partial slip, 2 = complete knot failure) $]$. $^{6-8}$

To assess participant stress during the transfer test, their physiological and perceived stress were measured. Physiological stress was assessed by capturing average heart rate (HR) and heart rate variability (HRV) with Polar H7 chest straps (Polar H7 HR monitor, Polar Electro Inc., Lake Success, NY) and the Elite HRV smart phone application (Elite HRV LLC). Perceived stress was assessed with the 6-item version of the STAI, which evaluates one's perception of their stress at a particular moment in time..$^{20}$

\section{Statistical Analyses}

Several multiple linear regressions with robust standard errors were performed, controlling for (i.e., also called holding the effect of other covariates fixed, ceteris paribus) race, age, gender, handedness, and number of training repetitions to consecutively assess the relationship between 
all baseline/post-test factors and transfer test suturing performance. In general, we predicted the transfer test suturing performance score using the following multivariable model:

$\hat{Y}_{i}=b_{0}+b_{1 i} X_{i}+b_{2}$ Age $+b_{3}$ Race $+b_{4}$ Gender $+b_{5}$ Handedness $+b_{6}$ Training repitions

Where, in each multivariable model i was consecutively automaticity, perceived ability in laparoscopic surgery, perceived ability on simulators, attentional selectivity, intracorporeal suturing performance, experience on simulators, and number of laparoscopic cases. If we hold all other covariates fixed (i.e., ceteris paribus), then the coefficient of the predictor variable tells us its effect on the dependent variable. An alternative to the multivariable method, which gives us the same estimate as above, is the use of a "Partialling Out" approach where we regress $Y_{i}$ on the residuals of the regression of $X_{i}$ on the covariates thus allowing us to estimate the effect of $X_{i}$ on $Y_{i}$ after the effect of all covariates on $X_{i}$ has been "netted out" or "partialled out". Training duration was not accounted for in baseline models.

We also assessed the assumptions of multiple linear regressions: normality using Shapiro-Wilk W test and constant variance of the error terms using Cameron \& Trivedi's decomposition of IM-test. ${ }^{21-23}$ If there was any indication of the violation of these assumptions, we used non-parametric bootstrapping methods to report the $95 \%$ confidence interval and the pvalue. In each consecutive multivariable model multicollinearity was also assessed using variance inflation factor for all the independent variables. ${ }^{24}$ The estimates from the multivariable analysis for each variable of interest in terms of change in transfer tests suturing performance score are reported as: coefficient ([95\% confidence interval], p-values). Paired t-tests were performed to assess any differences in significant predictor variables from baseline to post-test, and increases in stress from resting to transfer test performance. For all analyses, a p-value of 
$<0.05$ was considered significant. Data was analyzed with Stata/SE 14.2 statistical software (StataCorp. 2015. Stata Statistical Software: Release 14. College Station, TX).

\section{Results}

Data from 38 residents was analyzed. The average age of the participating residents was $29.7 \pm$ 3.7 years, there were $50 \%$ women, $73.7 \%$ general surgery residents, and the average postgraduate year of the participants was $2.1 \pm 1.1$ (Table 1). There was a significant increase in STAI-6 scores $(10.9$ vs. $13.3, \mathrm{p}<0.001)$ and HR (82.1 vs. $103.5, \mathrm{p}<0.001)$, and a significant decrease in HRV (53.5 vs. 42.0, p < 0.001) between resting and transfer test performance.

The results of the multiple linear regression revealed that ability to achieve automaticity during performance (i.e., assessed with the TOPS-3), perceived ability in laparoscopic surgery and on laparoscopic simulators, and attentional selectivity predicted transfer test suturing performance (Table 2). No other baseline factors predicted transfer test suturing performance. Regarding post-test results, only perceived ability in laparoscopic surgery and on simulators, and post-test suturing performance were significant predictors of transfer test suturing performance.

\section{Discussion}

In the current study, surgery residents' perceived ability to achieve automaticity at baseline, baseline attentional control, perceived ability in laparoscopic surgery and on simulators at baseline and post-test, and post-test suturing performance were predictive of skill transfer from the simulation laboratory to a stressful simulated clinical environment.

Unsurprisingly, participants' suturing performance on the simulator after training was predictive of suturing performance during the suturing transfer test. However, participants' baseline ability to achieve automaticity as assessed by the TOPS-3 instrument was the strongest predictor of skill transfer. Automaticity refers to one's ability to perform a task automatically without significant 
demands on one's attentional resources required to execute the movements, and is a known characteristic of expertise and has been previously shown to improve transfer of simulatoracquired laparoscopic skill to the clinical environment. ${ }^{13,25,26}$ The results of the present study support this finding, and suggest that trainee automaticity should not only be evaluated as an additional metric of laparoscopic surgical performance but also be used as a training goal as previously proposed. ${ }^{11}$ However, there may be additional factors related to automaticity that could impact learners' transfer of simulator-acquired skill that need to be considered.

Reinvestment theory posits that if a performer experiences heightened stress, automaticity processes can become disrupted. ${ }^{27,28}$ When automaticity is disrupted, some performers are prone to "reinvest" their attention on their movement, where they consciously think about movement execution, which can ultimately lead to performance failure. Researchers have found that in spite of achieving proficiency on the FLS peg transfer task on simulators, heightened stress during a performance test led students prone to reinvestment of attention to experience greater performance deteriorations than students with low propensity for reinvestment. ${ }^{29}$ Thus, reinvestment may be a factor that moderates surgical trainees' performance under heightened stress. Future studies investigating the relationship between automaticity and surgical performance should also consider trainees' propensity for reinvestment to help explain performance deterioration under heightened stress.

The findings from this study also support the use of the relevant TOPS subscale for the assessment of this skill. Specific items from the TOPS-3 assessing automaticity during performance include: "I can allow the whole skill or movement to happen naturally in a procedure without concentrating on each part", "I am able to perform skills in a procedure without having to consciously think about them", "In a procedure, I am sufficiently prepared to 
be able to perform on automatic pilot", and "I am able to trust my body to perform skills in a procedure".

Interestingly, resident's self-perceived ability with laparoscopy and simulators, which is reflective of self-efficacy, was also predictive of skill transfer but their prior clinical experience was not. Self-efficacy refers to one's belief in their capabilities to perform at a given performance standard and exert control over their choices, aspirations, effort, and engagement in a task, how long they persevere at a task in the face of challenge, the amount of stress they experience when coping with taxing environmental conditions, and whether their thoughts are helpful or disabling to their performance. ${ }^{30-32}$ Bandura $^{31}$ suggested that there are four primary antecedents of self-efficacy, which include: mastery experiences, verbal persuasion, physiological arousal, and vicarious experiences. Mastery experiences may be the most important source of information that individuals use to formulate their self-efficacy, but these experiences are not inherently developmental, rather, one needs to perceive that they are performing competently to facilitate increased self-efficacy. Thus, the resident's reported selfefficacy to perform laparoscopy may be more salient to performance than previous experience alone as it is also associated with increased self-confidence and ability to regulate one's practice and performance engagement. Therefore, while prior experience with laparoscopy and simulators was not significantly predictive of simulation-acquired skill transfer, higher perceived ability in these domains was.

Attentional selectivity (i.e., as assessed with the D2 test of attention) was also identified as a key element of residents' transfer of simulator-acquired skill to the clinical environment. This finding is congruent with previous research that has identified the ability to maintain selective attention and ignore distractions as an important characteristic of expert surgical 
performance. ${ }^{33}$ Indeed, there are numerous potential distractions in the operating room environment that compete for surgeons' attention, and a surgeon's ability to attend to taskrelevant stimuli is paramount to safe and effective surgical performance. Therefore, implementing strategies that enhance trainee's abilities to focus their attention and block out distractions may be valuable and should be considered during skills curricula design.

However, it is interesting to note that in spite of the observed relationship between attentional selectivity assessed by the D2 and transfer test performance, there was not a significant relationship between the TOPS-3 attentional control subscales and transfer test performance. We hypothesize that the global nature of the TOPS-3 (i.e., rating the frequency at which respondents use mental skills generally during practice and performance) may capture user's attention management generally, whereas the D2 assesses attentional selectivity, specifically. Thus, we feel that these instruments capture different aspects of attention. The identified predictors in this study can be useful to educators for skill assessment purposes and skills curriculum design. The identification of low scores in the domains of automaticity and attentional selectivity may indicate the need for additional training or increased emphasis of tasks that enhance these skills. In regards to self-efficacy, we recommend that surgical educators supplement skills training by regularly assessing residents' self-efficacy in addition to measures of technical proficiency to ensure "readiness". This is a more resource-intensive approach to resident training, but by assessing residents' self-efficacy to perform skills proficiently, individualized training can be better prescribed to ensure residents feel competent to perform specific skills.

Interestingly, we did not observe the ability of additional mental skills (i.e., aside from attentional selectivity and automaticity) to predict transfer test performance. We hypothesized 
that other mental skills, specifically mental imagery, would predict transfer test performance. In a recent systematic review on the application of mental skills in surgery, we found that several studies reported benefits of mental imagery use to skill acquisition and performance. ${ }^{10}$ Since these studies implemented mental imagery-specific training interventions, they taught learners how to effectively utilize mental imagery for surgical performance. In the present study, we only assessed learners' use of mental imagery for surgical practice and performance, so it is possible that participants utilized these skills ineffectively or improperly which ultimately limited their impact on their performance. Furthermore, even though other mental skills like goal setting and relaxation strategies have shown benefits to performance when applied in other domains, ${ }^{32}$ their use by the present study group did not predict transfer test performance. It is possible that in spite of the frequency of their use, participants' use of these and other mental skills may have been inadequate to truly benefit transfer test performance. Further study is needed to identify additional assessments related to the quality of learners' mental skills use in practice and performance settings to investigate this hypothesis.

One of the limitations of this study is the reliance on self-report assessment tools that introduce bias. Nevertheless, each instrument used was rigorously selected based on their known psychometric properties and utilization with high-performers within and outside of surgery. Data in the present study were also gathered from learners at two institutions, which may have introduced the risk for protocol consistency. However, the principal investigator and study coordinator were involved in all testing and training sessions at both institutions, and data gathering methodology followed a detailed protocol to ensure consistency across study sites. The findings from the study were obtained using parametric and non-parametric bootstrap multivariable regression analyses as appropriate. However, given the small sample of the 
residents in this study we recommend a future study involving more residents to substantiate these findings.

\section{Conclusions}

In conclusion, findings from this study indicate ability to achieve automaticity in performance, perceived ability in laparoscopic surgery and on laparoscopic simulators, and attentional selectivity, were significant predictors of simulator-acquired surgical skill transfer to the clinical environment. Assessing trainees' automaticity and attentional selectivity may help surgical educators identify residents who need additional training and develop more targeted interventions to enhance these domains, and ultimately, skill transfer. Also, in addition to technical skills assessments, regularly assessing trainees' self-efficacy to perform surgical skills may enable trainees to develop more skill-appropriate self-efficacy. This may help educators identify any "blind spots" in trainees' perceived ability and help them better align self-efficacy with technical skill.

\section{Acknowledgement}

The authors want to thank all volunteer participants at Indiana University School of Medicine and Carolinas Simulation Center, and all staff who participated in this study. 


\section{References}

1. Perone JA, Anton NE, Gardner AK, Steinemann S. Simulation Training in Surgical Education. Curr Surg Rep, 2017;5:20-31. doi:10.1007/s40137-017-0182-5

2. Nasca T, Day S, Jr EA. ACGME Duty Hour Task Force. The new recommendations on duty hours from the ACGME Task Force. N Engl J Med, 2010;3:1-6. doi:10.1056/NEJMsb1005800

3. Williams RG, Dunnington GL. Accreditation Council for Graduate Medical Education core competencies initiative: The road to implementation in the surgical specialties. Surg Clin North Am, 2004;84:1621-1646. doi:10.1016/j.suc.2004.06.018

4. Acton RD, Jones DB LKR. American College of Surgeons and Association for Surgical Education medical student simulation based surgical skills curriculum. Bull Am Coll Surg, 2015;100:35-39.

5. ACS/ASE/APDS Resident Prep Curriculum. 2014. https://www.facs.org/education/program/resident-prep. Accessed April 16, 2018.

6. Korndorffer Jr JR, Dunne JB, Sierra R, et al. Simulator training for laparoscopic suturing using performance goals translates to the operating room. J Am Coll Surg, 2005;201:23-29. doi: 10.1016/j.jamcollsurg.2005.02.021

7. Stefanidis D, Korndorffer Jr JR, Markley S, et al. Closing the gap in operative performance between novices and experts: does harder mean better for laparoscopic simulator training? $J$ Am Coll Surg, 2007;205:307-313. doi:10.1016/j.jamcollsurg.2007.02.080

8. Prabhu A, Smith W, Yurko Y, et al. Increased stress levels may explain the incomplete transfer of simulator-acquired skill to the operating room. Surgery 2010;147:640-645. doi:10.1016/j.surg.2010.01.007

9. Williams JM. Applied Sport Psychology. 6th ed, New York: McGraw-Hill, 2010.

10. Anton NE, Bean EA, Hammonds SC, Stefanidis D. Application of mental skills training in surgery: a review of its effectiveness and proposed next steps. J Laparoendosc Adv Surg Tech A, 2017;27:459-469. doi: 10.1089/lap.2016.0656

11. Stefanidis D, Scerbo MW, Korndorffer JR Jr, Scott DJ. Redefining simulator proficiency using automaticity theory. Am J Surg 2007;193:502-506.

12. Anton NE, Beane J, Yurco AM, et al. Mental skills training effectively minimizes operative performance deterioration under stressful conditions: Results of a randomized controlled study. Am J Surg. 2017. In press.

13. Stefanidis D, Scerbo MW, Montero PN, et al. Simulator training to automaticity leads to improved skill transfer compared with traditional proficiency-based training: a randomized controlled trial. Ann Surg, 2012;255:30-37. doi:10.1097/SLA.0b013e318220ef31

14. Hardy L, Roberts R, Thomas PR, Murphy SM. Test of performance strategies (TOPS): instrument refinement using confirmatory factor analysis. Psychol Sport Exerc, 2010;11:2735.

15. Arora S, Aggarwal R, Sevdalis N, et al. Development and validation of mental practice as a training strategy for laparoscopic surgery. Surg Endosc, 2010;24:179-187. doi: 10.1007/s00464-009-0624-y

16. Hall CR, Stevens DE, Paivio A. Sport imagery questionnaire: Test manual. Morgantown: West Virginia University, 2005.

17. Brickenkamp R, Zillmer E. The d2 test of attention. Cambridge: Hogrefe \& Huber Pub, 1998. 
18. Spielberger CD, Gorsuch RL, Lushene R, Vagg PR, Jacobs GA. Manual for the state-trait anxiety inventory. Palo Alto: Consulting Psychologists Press, 1983.

19. Society of American Gastrointestinal and Endoscopic Surgeons. Fundamentals of Laparoscopic Surgery (FLS). 2003-2008. Available at: http://www. flsprogram.org. Accessed April 23, 2018.

20. Bathalon S, Dorion D, Darveau S, Martin M. Cognitive skills analysis, kinesiology, and mental imagery in the acquisition of surgical skills. J Otolaryngol, 2005;34:328-332.

21. Shapiro SS, Wilk MB. An analysis of variance test for normality (complete samples). Biometrika, 1965;52:591-611.

22. Royston P. Shapiro-Wilk and Shapiro-Francia Tests. Stata Tech Bull, 1992;1:105.

23. Cameron AC, Trivedi PK. The information matrix test and its implied alternative hypotheses. 1990. Working paper 372, University of California-Davis, Institute of Governmental Affairs.

24. Chen X, Ender P, Mitchell M, Wells C. Regression with Stata, 2003. Available at: https://stats.idre.ucla.edu/stat/stata/webbooks/reg/default.htm. Accessed August $1^{\text {st }}, 2018$.

25. Stefanidis D, Scerbo MW, Korndorffer JR, Scott DJ. Redefining simulator proficiency using automaticity theory. Am J Surg, 2007;193:502-506. doi: 10.1016/j.amjsurg.2006.11.010

26. Stefanidis D, Scerbo MW, Sechrist C, et al. Do novices display automaticity during simulator training? Am J Surg, 2008;195:210-213. doi: 10.1016/j.amjsurg.2007.08.055

27. Masters RS. Knowledge, knerves and know $\square$ how: The role of explicit versus implicit knowledge in the breakdown of a complex motor skill under pressure. Br J Psychol, 1992;83:343-358.

28. Masters R, Maxwell J. The theory of reinvestment. Int Rev Sport Exerc Psychol, 2008;1:160183.

29. Malhotra N, Poolton JM, Wilson MR, Ngo K, Masters RS. Conscious monitoring and control (reinvestment) in surgical performance under pressure. Surg Endosc, 2012;26:2423-2429. doi: 10.1007/s00464-012-2193-8

30. Bandura A. Self-efficacy: Toward a unifying theory of behavioral change. Psychol Rev, 1977;84:191-215.

31. Bandura A. Social foundations of thought and action: A social cognitive theory. Englewood Cliffs: Prentice-Hall, Inc, 1986.

32. Bandura A. Social cognitive theory of self-regulation. Organ Behav Hum Decis, 1991;50:248-287.

33. Arora S, Sevdalis N, Nestel D, et al. The impact of stress on surgical performance: a systematic review of the literature. Surgery, 2010;147:318-330. doi:

10.1016/j.surg.2009.10.007 
Table 1: Characteristics of the study sample

\begin{tabular}{|c|c|c|}
\hline Characteristics & In sample $(\mathrm{n}=38)$ & p-value \\
\hline \multicolumn{3}{|l|}{ Race $(\mathrm{n}, \%)$} \\
\hline Non-white & $13(34.21)$ & \\
\hline White & $21(55.26)$ & \\
\hline No information & $4(10.53)$ & \\
\hline Age (mean, SD) & $29.66(3.71)$ & \\
\hline \multicolumn{3}{|l|}{ Handedness $(\mathrm{n}, \%)$} \\
\hline Right & $34(89.47)$ & \\
\hline Left & $2(5.26)$ & \\
\hline Ambidextrous & $2(5.26)$ & \\
\hline \multicolumn{3}{|l|}{ Gender $(\mathrm{n}, \%)$} \\
\hline Male & $19(50.00)$ & \\
\hline Female & $19(50.00)$ & \\
\hline \# training repetitions (mean, SD) & $29.37(8.17)$ & \\
\hline $\begin{array}{l}\text { Trainee characteristics of interes } \\
\text { posttest }\end{array}$ & & \\
\hline \multicolumn{3}{|c|}{ Autuomaticity in Performance (mean,SD) } \\
\hline Baseline & $2.87(0.59)$ & 0.0037 \\
\hline Posttest & $3.15(0.60)$ & \\
\hline \multicolumn{3}{|c|}{ Perceived Ability in Laparoscopic Surgery (mean,SD) } \\
\hline Baseline & $6.68(3.89)$ & $<0.001$ \\
\hline Posttest & $10.29(3.22)$ & \\
\hline \multicolumn{3}{|c|}{ Perceived Ability on Simulators (mean,SD) } \\
\hline Baseline & $7.92(3.92)$ & 0.0002 \\
\hline Posttest & $10.58(3.43)$ & \\
\hline \multicolumn{3}{|l|}{ Attentional Selectivity (mean,SD) } \\
\hline Baseline & $223.16(40.04)$ & $<0.001$ \\
\hline Posttest & $244.24(34.37)$ & \\
\hline \multicolumn{3}{|c|}{ Intracorporeal Suturing Performance (mean,SD) } \\
\hline Baseline & $157.54(151.34)$ & $<0.001$ \\
\hline Posttest & $355.16(133.95)$ & \\
\hline \multicolumn{3}{|c|}{ Experience on Simulators (mean,SD) } \\
\hline Baseline & $8.93(11.32)$ & \\
\hline \multicolumn{3}{|l|}{ \# Laparoscopic Cases (mean,SD) } \\
\hline Baseline & $20.21(27.25)$ & \\
\hline
\end{tabular}


Table 2: Multivariable analyses of the factors at baseline and post-test predicting transfer test performance

\begin{tabular}{lrrrrrr}
\hline \multicolumn{1}{c}{ Factors $^{\#}$} & \multicolumn{3}{c}{ Baseline } & \multicolumn{3}{c}{ Posttest } \\
\cline { 2 - 7 } & Coefficient & $95 \%$ CI & p-value & Coefficient & 95\% CI & p-value \\
\hline Automaticity in Performance & 83.55 & {$[15.57,151.53]$} & 0.018 & 27.09 & {$[-72.79,126.97]^{*}$} & 0.595 \\
Perceived Ability in Laparoscopic & 16.11 & {$[5.90,26.32]$} & 0.003 & 15.68 & {$[4.47,26.90]$} & 0.008 \\
Surgery & 15.05 & {$[4.96,25.15]$} & 0.005 & 14.07 & {$[3.31,24.82]$} & 0.012 \\
Perceived Ability on Simulators & 1.37 & {$[0.33,2.42]$} & 0.012 & 1.18 & {$[-0.21,2.57]$} & 0.093 \\
Attentional Selectivity & 0.3 & {$[-0.05,0.65]$} & 0.092 & 0.35 & {$[0.09,0.60]$} & 0.009 \\
Intracorporeal Suturing & 2.83 & {$[-0.42,6.07]$} & 0.085 & & & \\
Performance & 1.14 & {$[-0.12,2.40]$} & 0.075 & & & \\
Experience on Simulators & & & & & \\
\# Laparoscopic Cases & & &
\end{tabular}

*Boot-strap standard error and 95\% CI for non-parametric regression

${ }^{\#}$ Each factor was consecutively used in separate multivariable models after adjusting for the effect of age, race, sex, and handedness. In addition, for the posttest models we also controlled for the number of training repetitions 
Figure 1. Study Algorithm

\section{Baseline Testing}

FLS Peg Transfer, Pattern Cut, Intracorporeal Suturing

Test of Performance Strategies- Version 3 (TOPS-3)

D2 Test of Attention (D2)

Mental Imagery Questionnaire (MIQ)

Sport Imagery Questionnaire (SIQ)

State-Trait Anxiety Inventory- Full Version (STAI-F)

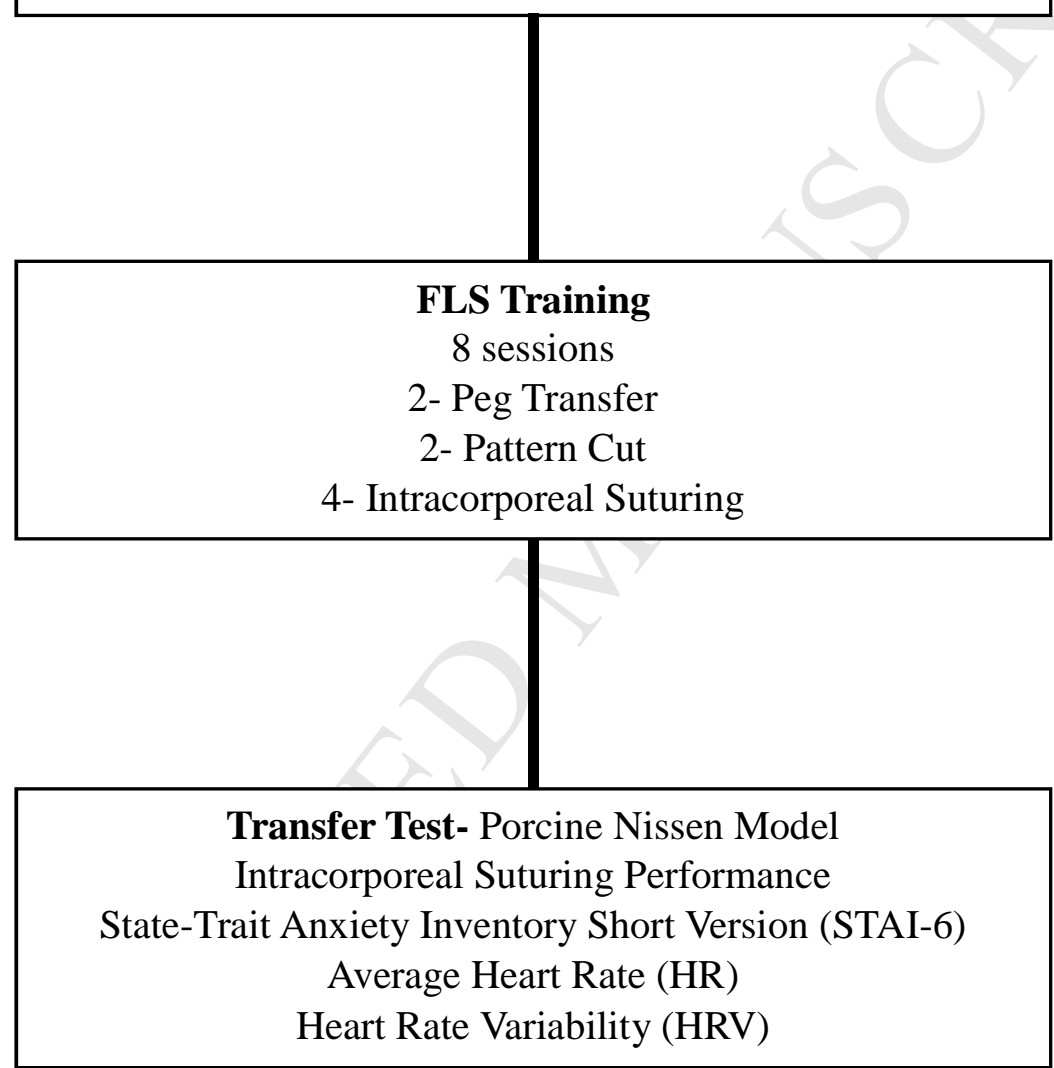




\section{Highlights}

- Automaticity and attentional selectivity predict the transfer of skill

- Perceived ability in laparoscopic surgery was predictive of skill transfer

- Perceived ability on laparoscopic simulators was predictive of skill transfer

- Focusing on these factors may facilitate more complete skill transfer for trainees 